\title{
Original COST EFFECTIVENESS ANALYSIS OF LETROZOLE COMPARED WITH Article TAMOXIFEN AS INITIAL ADJUVANT THERAPY FOR POSTMENOPAUSAL WOMEN WITH ENDOCRINE RESPONSIVE BREAST CANCER
}

\author{
Naser Abd El Bary ${ }^{1}$, Mina Maher ${ }^{1}$, Mohamed Elsherbini ${ }^{1}$, Mohamed Abdeen ${ }^{2}$ and \\ Tarek Hashem ${ }^{1}$ \\ ${ }^{1}$ Clinical Oncology Department, Faculty of Medicine, Menoufia University, Shibin El-Kom, Egypt; \\ 2 Clinical Oncology Department, Faculty of Medicine, Cairo University, Cairo, Egypt
}

\begin{abstract}
Background: There is much excitement in the oncology community with the recent emergence of a variety of new treatments like aromatse inhibitors and molecular targeted therapies. Such treatments, however, come at considerable cost. Economic studies involve a comparison of the costs and benefits of alternative treatment options to ensure that the money spent will provide its highest value and this, of course, will aid decision makers to direct resources properly. Aim of the Work: To assess the cost-effectiveness of letrozole compared with tamoxifen as Initial adjuvant therapy for postmenopausal women with endocrine-sensitive breast cancer in Egypt.

Materials and Methods: The methods for this evaluation were based on the Canadian model that was adapted to calculate the incremental cost effectiveness ratio (ICER) between upfront tamoxifen and upfront letrozole (femara) in postmenopausal women with breast cancer using Markov state transition model with an Egyptian healthcare system cost perspective. We applied the model and calculated such data on 104 patients at our institute. Since there is no wellestablished threshold for the ICER in Egypt, we used WHO-CHOICE (CHOosing Interventions that are Cost-Effective) guidelines that correlate the intervention to the national income per capita.

Results: ICER was calculated by two methods: the primary analysis) according to DFS event rate) and was 3471.63 USD/QALY and by a secondary analysis (according to TTR event rate) it was 4493.72 USD/ QALY. The first analysis showed that ICER is very cost effective (costs QALY is less than the national income per capita which is less than 3940 USD in Egypt(, while the second analysis showed that it is cost effective.

Updating this result according to the incidence of DFS events published in BIG 176(98- months analysis) the ICER is 5312.3 USD/QALY and still cost effective.

Conclusion: Although letrozole (femara) seems to be expensive in comparison to tamoxifen, comprehensive evaluation of total drug costs and the gained benefits proved the superiority of femara. Awareness of decision makers with this data is of an utmost importance for the direction of budget especially in areas of limited resources such as Egypt.
\end{abstract}

Key Words: Cost effectiveness analysis, Letrozole, Tamoxifen, Adjuvant

Corresponding Author: Naser Abd El Bary Tel.:0105442335

\section{INTRODUCTION}

The rapid progress in diagnosis and treatments of cancer leads to introduction of a variety of diagnostics and treatments in oncology. Many of these interventions are costly; this increases the burden on the patients and society $^{1}$. Cost-effectiveness studies compare between the costs and the effectiveness of alternative treatment options. These help decision makers to allocate a fixed health budget between interventions to achieve the maximum health benefits from the minimal costs ${ }^{2}$.

Cost is measured in monetary units such as pounds sterling or US dollars. While benefits are usually expressed in quality-adjusted life years QALYs ${ }^{3}$. QALY takes into account both quantity and the quality of life generated by healthcare interventions. It is the arithmetic product of life expectancy and a measure of the quality of the remaining life years.

Proper assessment of the costs in medical economic studies should include:
1. Direct treatment cost: the cost of resources used by health sector to provide treatment (e.g. cost of hospital stay, cost of chemotherapy, chemotherapy administration cost, overhead).

2. Indirect treatment costs: including costs of resources used by the patients and their families to access and participate in treatment such as traveling and parking as well as lost work time incurred by the patient and their families.....etc ${ }^{1}$. Cost-effectiveness is typically expressed as an incremental cost-effectiveness ratio (ICER). The ICER is the ratio between the difference in costs and the difference in benefits of two interventions, as given by the following formula:

$$
\mathrm{CE}=\frac{\mathrm{C} 2-\mathrm{C} 1}{\mathrm{E} 2-\mathrm{E} 1}
$$

Where $\mathrm{C}$ represents cost of each intervention and $\mathrm{E}$ represents effectiveness, regimen 2 is the new regimen 
and regimen 1 is the comparator ${ }^{2}$. A threshold value is often set by policy makers, who may decide that only interventions with an ICER below the threshold are cost effective. In the United Kingdom, as of January 2005, the National Institute for Health and Clinical Excellence NICE is believed to have a threshold of about 30,000 pounds sterling per QALY ${ }^{4}$.

In USA, 50,000 United States dollars USD per QALY is often suggested as a threshold ICER for a cost-effective intervention $^{3}$. Canadian authors have proposed that interventions costing less than 20,000 Canadian dollars per QALY be considered cost-effective ${ }^{5}$. These cut points are arbitrary. WHO-CHOICE (choosing Interventions that are Cost-Effective) has been using criteria suggested by the Commission on Macroeconomics and Health; Geneva 2001: interventions that provide one QALY for less than average per capita income for a given country or region are considered very cost-effective; interventions that cost less than three times average per capita income per QALY gained are still considered cost-effective; and those that exceed this level are considered not costeffective ${ }^{6}$.

According to the latest available data from the World Bank the gross national income (GNI) per capita at purchasing power parity (PPP) in Egypt for the year 2003 is 3940.00 \$ United States Dollars ${ }^{7}$.

Tamoxifen has been the predominant first line hormonal therapy for breast cancer ${ }^{8}$. Five years of adjuvant tamoxifen is associated with $47 \%$ annual reduction in the recurrence rate and $26 \%$ annual reduction in the death rate $^{9}$. However third generation aromatase inhibitors such as letrozole represent a significant advantage in terms of improved efficacy and tolerability and now being considered an alternative to tamoxifen as first line ${ }^{8}$.

According to the results of BIG 1-98 (51 months follow up) letrozole had a significant $19 \%$ improvement in DFS compared with those receiving tamoxifen. Distant metastases accounted for the majority of DFS events in both the letrozole and tamoxifen groups. Initial adjuvant letrozole therapy resulted in a significant $27 \%$ reduction in the risk of distant metastases when compared with tamoxifen and this benefit was irrespective of the site of distant metastases. Also at this early stage of follow-up, a $14 \%$ improvement in OS was observed with letrozole, but the difference was not significant $(P=0.16)$ till the time of this study ${ }^{10}$.

Update of this data at a median 76 months of followup, five years of letrozole monotherapy increased survival $13 \%$ in the intent-to-treat analysis $(\mathrm{P}=0.08)$ and a significant $19 \%$ in an analysis censored for extensive "contamination" by off-protocol switching from tamoxifen to letrozole and associated with the following in comparison to tamoxifen monotherapy:

1. Improved overall survival (HR 0.87 intent to treat, $\mathrm{P}=0.08$ and 0.81 censored, $95 \%$ confidence interval 0.69 to 0.94 ).

2. Disease-free survival (HR 0.88 intent to treat, $\mathrm{P}=0.03$ and 0.84 censored, $95 \%$ CI 0.74 to 0.95 ).

3. Time to distant recurrence (HR 0.85 intent to treat, $\mathrm{P}=0.05$ and 0.81 censored, $95 \%$ CI 0.68 to 0.96$)^{11}$.

\section{MATERIALS AND METHODS}

The methods for this evaluation were based on those developed for a Canadian evaluation of upfront anastrazole, sequential tamoxifen-exemestane and extended tamoxifen-letrozole by C. Skedgel et al. ${ }^{12}$. The Canadian model was adapted to calculate the incremental cost effectiveness ratio ICER between upfront tamoxifen and upfront letrozole in postmenopausal women with breast cancer and an Egyptian healthcare system cost perspective.

A Markov state transition model was developed reproducing the natural history of breast cancer excluding carcinoma in situ to predict the cumulative incidencebased costs and QALYs gains for a hypothetical cohort of 1000 postmenopausal women with hormone receptorpositive breast cancer who had undergone curative surgery and undergoing adjuvant hormonal therapy similar to that used by $\mathrm{T}$ Younis et al. ${ }^{13}$.

The model incorporated five health states (Figure 1): well on treatment, well off treatment, in local relapse (combining locoregional and contralateral recurrences), in distant relapse and dead (with or without relapse). Adverse side-effects (ASEs) included in the model were vaginal bleeding (endometrial hyperplasia) and endometrial carcinoma, venous thromboembolism and bone fractures. The DFS event rate, TTR event rate and incidence of ASEs were included from the literature based on the basis the published results of BIG 1-98 trial (51 months follow up) in the literature. For purposes of calculating QALYs, each health state was associated with a quality-of-life weight ranging from 1.0 (perfect health) to 0.0 (death). Quality weights were derived from the literature. The generated cumulative costs and QALY gains were calculated in 10 fixed time periods of 1 year.

Retrospective chartreview, a total of 104 patients'charts from Menofia University Hospital Oncology Department was reviewed in the period between April 2007 and April 2008. Only direct medical costs was calculated including the following: Cost of visits, cost of endocrine therapy, cost of chemotherapy, cost of radiotherapy, cost of surgical interference, cost of other drugs e.g. analgesics, antibiotics, drugs for gastritis, granulocyte colony stimulating factor, calcitonin, calcium, bisphosphonates..........etc., cost of imaging, cost of laboratory tests, tumor markers, pathology and cost of hospital admission as done in the study of V. Cocquyt et al. ${ }^{14}$. Both future costs and lifeyears were also discounted at a $3 \%$ yearly rate to account for time preference ${ }^{15}$. Multi-way sensitivity analyses are 
undertaken which re-estimate cost-effectiveness when one or more input parameters are altered ${ }^{8}$. In the primary analysis the effectiveness of both drugs was based on the relative hazard ratio of DFS among women with hormonal receptor positive $(\mathrm{ER}+$ and $\backslash$ or $\mathrm{PR}+)$ breast cancer. While TTR hazards were applied in a secondary analysis.

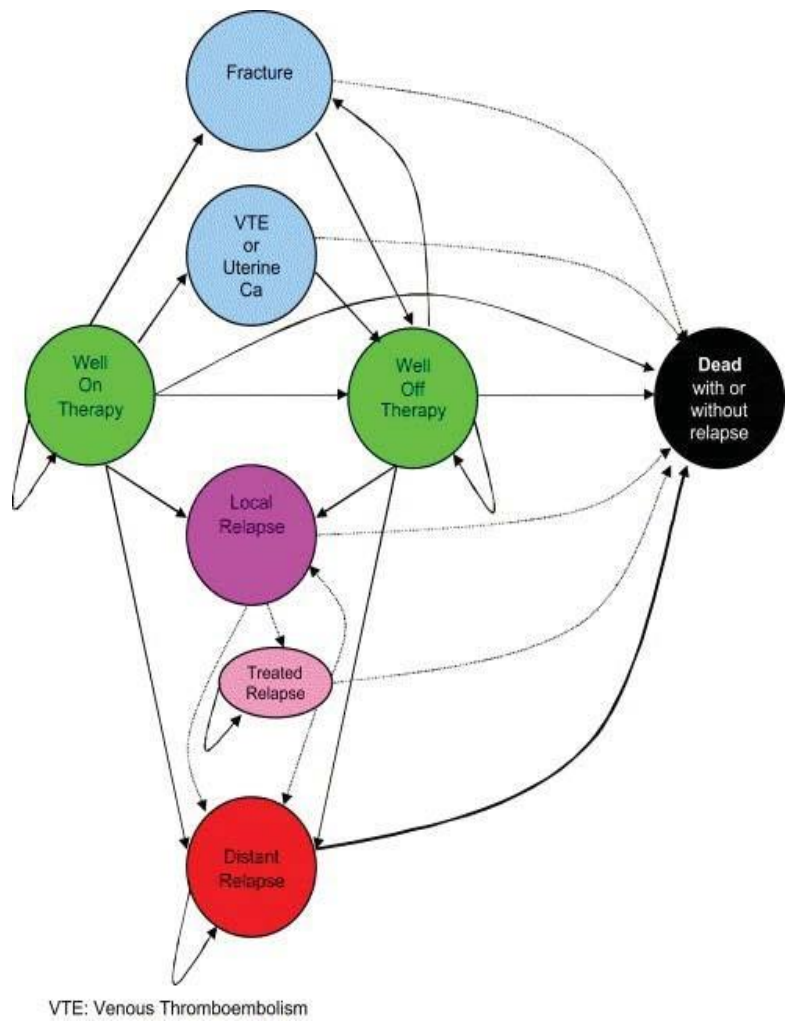

Figure 1: Model schematic ${ }^{12}$ (C. Skedgel et al.).

\section{RESULTS}

One hundred and four patients from Menofia Oncology Hospital were evaluated for the study. Table (1) shows patient characteristics at presentation.

Table (2) shows the Prediction of 10 years cumulative cost of breast cancer treatment in a hypothetical cohort of 1000 patients in both tamoxifen and letrozole groups according to DFS events and incidence of complication in literature based on results of BIG 1-98 trial, showing that the total costs in tamoxifen group is 2203177.1 USD versus 3680353.6 USD in letrozole group. The higher costs in letrozole are mainly due to the high costs of the drug in patients well on treatment and higher incidence of bone fractures in this group. This also may be compensated by the higher costs of treatment of relapse as well as complications of tamoxifen.

Distribution of costs among different health states in both letrozole and tamoxifen groups according to DFS events is shown in Figure (2) that summarizes the results shown in Table (2).

The predicted 10 years costs of hypothetical cohort of 1000 patients in both groups according to incidence of TTR events and complications is shown in Table 3 showing that the total costs in tamoxifen group is 2259159.4 USD versus 4162161.8USD in letrozole group due to higher incidence of TTR events in letrozole group 54.13 versus 44.86 in tamoxifen group.

Distribution of costs among different health (Markov) states in both letrozole and tamoxifen groups according to TTR events is shown in Figure (3) that summarizes the results shown in Table(3).

The predicted 10 years QALYs in disease free patients in a hypothetical cohort of 1000 patients in both groups according to incidence of DFS events and complications incidence shown in Table (4) revealing that QALYs gained in letrozole group are 4986.92 QALYs in comparison to 4986.92 QALYs in tamoxifen group the difference is due to higher incidence of DFS events in letrozole group and better quality of life in the same group due to lower incidence of complications and relapse.

Distribution of the QALYs among disease free patients in a hypothetical cohort of 1000 patients in both letrozole and tamoxifen groups according to DFS events rate is shown in Figure (4) summarizing Table (4).

The predicted 10 years QALYs among disease free patients in a hypothetical cohort of 1000 patients in both groups according to incidence of TTR events and complications incidence is shown in Table 5 revealing that QALYs gained in letrozole group are 5722.31 QALYs in comparison to 5298.83QALYs in tamoxifen group.

Distribution of the QALYs among disease free patients in a hypothetical cohort of 1000 patients in both letrozole and tamoxifen groups according to TTR events rate is shown in Figure (5) that summarizes Table (5).

Assessment of incremental cost-effectiveness ratio ICER in USD/QALY using simple statistical analysis methods:

1. In the primary analysis (according to DFS event rate).

$$
\mathrm{ICER}=\frac{\mathrm{C} 2-\mathrm{C} 1 \quad 3680353.6-2203177.1}{\mathrm{E} 2-\mathrm{E} 1 \quad 4986.92-4561.42}=3471.63
$$

2. In the secondary analysis (according to TTR event rate).

$$
\mathrm{ICER}=4493.72
$$

Because cost-effectiveness in general is given in US dollars (USD) in the literature, costs were converted to this currency using the average exchange rate for the $3^{\text {rd }}$ December 2009 USD equals 5.445 Egyptian bounds. Since there is no well-established threshold for the ICER in Egypt, we will go with the WHO-CHOICE guide lines that determine that the intervention that costslQALY is less than the annual national income per capita is very cost-effective (in Egypt less than 3940.00USD). 
While the intervention that costs\QALY is less than three times national income per capita (in Egypt less than 11820.00 USD) is cost-effective.

So that, in this study in the primary analysis upfront letrozole in postmenopausal women is very cost-effective than tamoxifen (the previous standard treatment). While in the secondary analysis upfront letrozole in postmenopausal women is cost-effective than tamoxifen.

Updating this study according to the incidence of DFS events published in BIG 1-98 (76 months analysis), 509 DFS events in letrozole group versus 565 events in tamoxifen group.

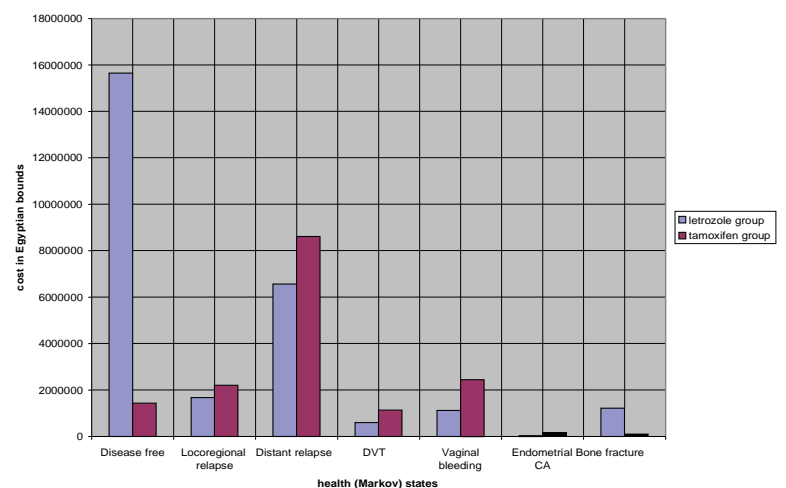

Figure 2: Distribution of costs among different health states in both letrozole and tamoxifen groups according to DFS events:

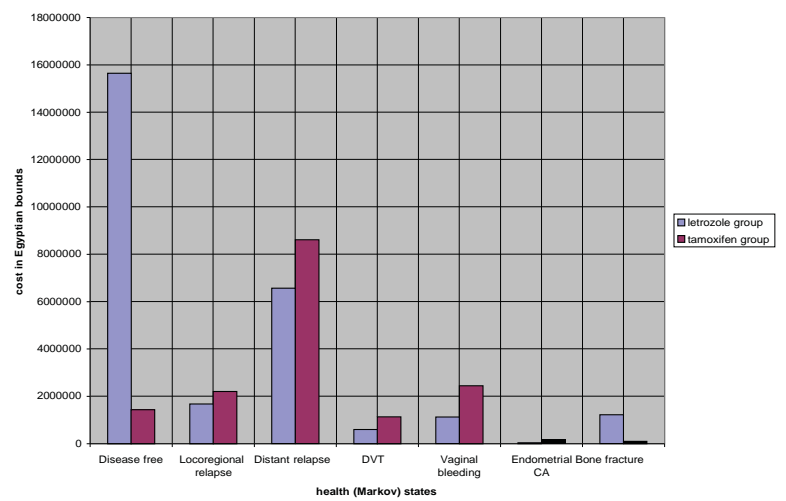

Figure 3: Distribution of costs among different health (Markov) states in both letrozole and tamoxifen groups according to TTR events:

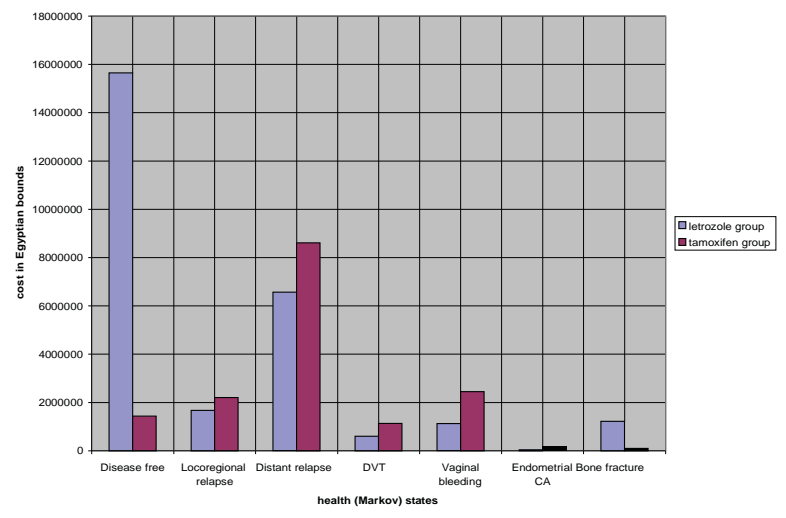

Figure 4: Distribution of the QALYs among disease free patients in a hypothetical cohort of 1000 patients in both letrozole and tamoxifen groups according to DFS events rate:
The predicted 10 years costs of hypothetical cohort of 1000 patients in both groups according to the new incidence of DFS events and complications is shown in Table (6).

The predicted 10 years QALYs in disease free patients in a hypothetical cohort of 1000 patients in both groups according to incidence of DFS events and complications incidence is shown in Table (7).

ICER was calculated (according to DFS event rate) $=5312.3 \mathrm{USD} / \mathrm{QALY}$.

So that upfront letrozole in postmenopausal women is still cost-effective than tamoxifen.

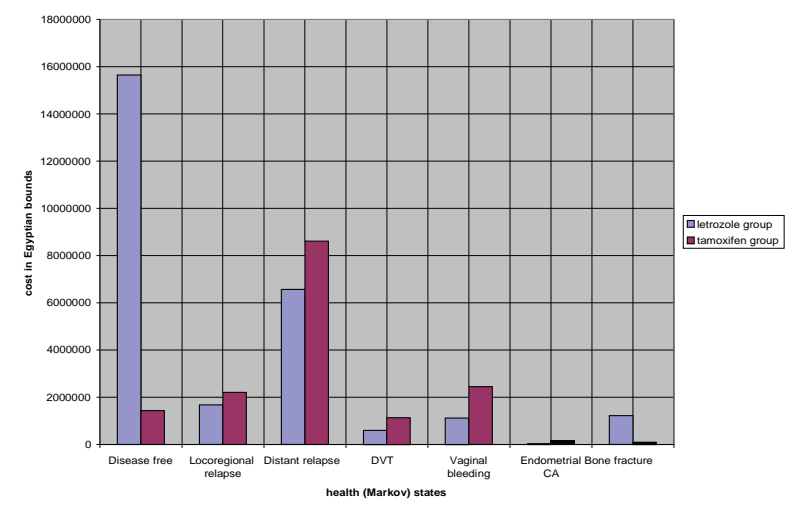

Figure 5: Distribution of the QALYs among disease free patients in a hypothetical cohort of 1000 patients in both letrozole and tamoxifen groups according to TTR events rate:

Table 1: patient characteristics at presentation.

\begin{tabular}{|c|c|}
\hline Age & $\begin{array}{l}\text { Range } 42-75 \text { years } \\
\text { Mean } 57.38 \text { years } \\
\text { SD } 7.055\end{array}$ \\
\hline Surgery & $\begin{array}{l}\text { MRM } 101 \text { patients } \quad(97 \%) \\
\text { Conservative surgery } 3 \text { patients }(3 \%)\end{array}$ \\
\hline Tumor size $\quad T$ & $\begin{array}{ll}\text { T1 } 12 \text { patients } & (11.5 \%) \\
\text { T2 70 patients } & (67.3 \%) \\
\text { T3 22 patients } & (21.2 \%)\end{array}$ \\
\hline Lymph node $\mathrm{N}$ & $\begin{array}{ll}\mathrm{N}-\text { ve } 31 \text { patients } & (30.8 \%) \\
\mathrm{N} \leq 332 \text { patients } & (30.8 \%) \\
\mathrm{N}>340 \text { patients } & (38.4 \%)\end{array}$ \\
\hline $\begin{array}{l}\text { Estrogen receptor } \\
\text { ER +ve }\end{array}$ & 95 patients \\
\hline $\begin{array}{l}\text { Progesterone receptor } \\
\text { PR +ve }\end{array}$ & 76 patients \\
\hline HER2/neu & $\begin{array}{c}\text { HER2/neu +ve } 23 \text { patients }(22.1) \\
\text { HER2/neu -ve } 24 \text { patients }(23.1 \%) \\
\text { HER2/neu unknown } 57 \text { patients }(54.8 \%)\end{array}$ \\
\hline $\begin{array}{l}\text { Adjuvant } \\
\text { chemotherapy }\end{array}$ & $\begin{array}{lc}\text { FAC 71 patients } & (68.3 \%) \\
\text { FEC 11 patients } & (10.6 \%) \\
\text { CMF 6 patients } & (5.8 \%) \\
\text { AC 5 patients } & (4.8 \%) \\
\text { EC 1 patient } & (0.9 \%) \\
\text { No chemotherapy } & (9.6 \%)\end{array}$ \\
\hline Adjuvant radiotherapy & 87 patients received RTX (84\%) \\
\hline
\end{tabular}


Table 2: the Prediction of 10 years cumulative cost of breast cancer treatment in a hypothetical cohort of 1000 patients in both tamoxifen and letrozole groups according to DFS and TTR event rates and incidence of complication in BIG 1-98 trial.

\begin{tabular}{lcccc}
\hline \multirow{2}{*}{ Markov (health) state } & \multicolumn{2}{c}{ TAM (C1) } & LET (C2) \\
\cline { 2 - 5 } Well on treatment & Incidence \% & Cost per 1000 & Incidence \% & Cost per 1000 \\
\hline Locoregional relapse & 34.9 & 1431266.50 & 44.2 & 15647684.00 \\
\hline Distant relapse & 8.3 & 2204205.30 & 6.3 & 1673071.50 \\
\hline DVT & 18.5 & 8609185.90 & 14.1 & 6561595.70 \\
\hline Vaginal bleeding & 3.8 & 1130128.00 & 2.0 & 594804.20 \\
\hline Endometrial CA & 8.3 & 2444789.10 & 3.8 & 3719301.00 \\
\hline Bone fracture & 0.65 & 152531.47 & 0.16 & 1218961.80 \\
\hline Total cost in Egyptian Bounds & 15.2 & 102934.55 & 18 & 26852964.00 \\
\hline Total cost in USD & & 16075041.00 & 4931673.8 \\
\hline 3\% discounted cost & 2952257.3 & & 3680353.6 \\
\hline
\end{tabular}

Table 3: The predicted 10 years costs of hypothetical cohort of 1000 patients in both groups according to incidence of TTR events and complications.

\begin{tabular}{lcccc}
\hline \multirow{2}{*}{ Markov (health) state } & \multicolumn{2}{c}{ TAM (C1) } & LET (C2) \\
\cline { 2 - 5 } & Incidence \% & Cost per 1000 & Incidence \% & Cost per 1000 \\
\hline Well on treatment & 44.86 & 1839731.00 & 54.13 & 19163100.30 \\
\hline Locoregional relapse & 8.3 & 2204205.30 & 6.3 & 1673071.50 \\
\hline Distant relapse & 18.5 & 8609185.90 & 14.1 & 6561595.70 \\
\hline DVT & 3.8 & 1130128.00 & 2.0 & 594804.20 \\
\hline Vaginal bleeding & 8.3 & 2444789.10 & 3.8 & 1119301.00 \\
\hline Endometrial CA & 0.65 & 152531.47 & 0.16 & 1218961.80 \\
\hline Bone fracture & 15.2 & 102934.55 & 18 & 30368381.00 \\
\hline Total cost in Egyptian Pounds & & 16483505.00 & & 5577296.8 \\
\hline Total cost in USD & & 3027273.6 & & 4162161.8 \\
\hline 3\% discounted cost & 2259159.4 & & \\
\hline
\end{tabular}

Table 4: The Prediction of 10 years cumulative QALYs gained by the disease free patients (Effectiveness) in a hypothetical cohort of 1000 patients in both tamoxifen and letrozole groups according to DFS and TTR event rates and incidence of complication in BIG 1-98 trial:

\begin{tabular}{lcccc}
\hline \multirow{2}{*}{ Patient state } & \multicolumn{2}{c}{ TAM (E1) } & \multicolumn{2}{c}{ LET (E2) } \\
\cline { 2 - 5 } & Incidence \% & QALYs per 1000 & Incidence \% & QALYs per 1000 \\
\hline Well on treatment & 34.9 & 3472.55 & 44.2 & 4397.9 \\
\hline Patient with DVT & 3.8 & 372.02 & 2.0 & 195.8 \\
\hline Patient with vaginal bleeding & 8.3 & 823.36 & 3.8 & 18 \\
\hline Patient with bone fracture & 15.2 & 1462.24 & 6130.17 & 6702 \\
\hline Total & & 4561.42 & & 4986.92 \\
\hline 3\% discounted total QALYs & & & 47.6 \\
\hline
\end{tabular}

Table 5: The predicted 10 years QALYs among disease free patients in a hypothetical cohort of 1000 patients in both groups according to incidence of TTR events and complications incidence:

\begin{tabular}{lcccc}
\hline \multirow{2}{*}{ Patient state } & \multicolumn{2}{c}{ TAM (E1) } & \multicolumn{2}{c}{ LET (E2) } \\
\cline { 2 - 5 } & Incidence \% & QALYs per 1000 & Incidence \% & QALYs per 1000 \\
\hline Well on treatment & 44.86 & 4463.57 & 54.13 & 5385.94 \\
\hline Patient with DVT & 3.8 & 372.02 & 2.0 & 195.8 \\
\hline Patient with vaginal bleeding & 8.3 & 823.36 & 3.8 & 18 \\
\hline Patient with bone fracture & 15.2 & 1462.24 & 376.96 \\
\hline Total & & 7121.19 & 7690.3 \\
\hline 3\% discounted total QALYs & 5298.83 & & 5722.31 \\
\hline
\end{tabular}


Table 6: The predicted 10 years costs of hypothetical cohort of 1000 patients in both groups according to incidence of DFS events and complications:

\begin{tabular}{|c|c|c|c|c|}
\hline \multirow{2}{*}{ Markov (health) state } & \multicolumn{2}{|c|}{ TAM (C1) } & \multicolumn{2}{|c|}{ LET (C2) } \\
\hline & Incidence $\%$ & Cost per 1000 & Incidence \% & Cost per 1000 \\
\hline Well on treatment & 36.4 & 1492782.2 & 43.6 & 15435272.0 \\
\hline Locoregional relapse & 8.3 & 2204205.30 & 6.3 & 1673071.50 \\
\hline Distant relapse & 18.5 & 8609185.90 & 14.1 & 6561595.70 \\
\hline DVT & 3.8 & 1130128.00 & 2.0 & 594804.20 \\
\hline Vaginal bleeding & 8.3 & 2444789.10 & 3.8 & 1119301.00 \\
\hline Endometrial CA & 0.65 & 152531.47 & 0.16 & 37546.21 \\
\hline Bone fracture & 15.2 & 102934.55 & 18 & 1218961.80 \\
\hline Total cost in Egyptian Bounds & & 16136556.52 & & 26640552.0 \\
\hline Total cost in USD & & 2963554.92 & & 4892663.36 \\
\hline $3 \%$ discounted cost & & 2211608.15 & & 3651241.31 \\
\hline
\end{tabular}

Table 7: The predicted 10 years QALYs in disease free patients in a hypothetical cohort of 1000 patients in both groups according to incidence of DFS events and complications incidence:

\begin{tabular}{lcccc}
\hline \multirow{2}{*}{ Patient state } & \multicolumn{2}{c}{ TAM (E1) } & \multicolumn{2}{c}{ LET (E2) } \\
\cline { 2 - 5 } & Incidence \% & QALYs per 1000 & Incidence \% & QALYs per 1000 \\
\hline Well on treatment & 36.4 & 3621.8 & 43.6 & 4338.2 \\
\hline Patient with DVT & 3.8 & 372.02 & 2.0 & 3.8 \\
\hline Patient with vaginal bleeding & 8.3 & 823.36 & 18 & 376.96 \\
\hline Patient with bone fracture & 15.2 & 1462.24 & 1731.6 \\
\hline Total & & 6279.42 & 6642.56 \\
\hline 3\% discounted total QALYs & & 4686.13 & 4957.13 \\
\hline
\end{tabular}

\section{DISCUSSION}

Tamoxifen (Nolvadex) roughly costs $462.00 £$ per year in comparison to letrozole (Femara) which costs $6660.00 £$ per year, so that letrozole seems to be very expensive in comparison to tamoxifen. This is not accurate because the economic studies should calculate both direct and indirect treatment costs.

The difference in the costs between letrozole and tamoxifen is compensated by increased incidence of locoregional recurrence and distant recurrence in tamoxifen group. Also increase incidence of DVT, vaginal bleeding and endometrial carcinoma leads to increase of the total cost of tamoxifen and thus decreasing the gap between the price of letrozole and tamoxifen. The increase in hip fracture rate in letrozole group was smaller, so that does not increase the gap between letrozole and tamoxifen so much.

The results of this study are consistent with other published studies comparing AI alternatives to TAM alone. A review by Karnon J. et al. ${ }^{8}$ found 7 unique economic evaluations comparing adjuvant AI strategies to TAM alone. Four evaluations of upfront AI relative to TAM reported cost-utilities in the range of CDN\$20,00032,000 per QALY gained, while one reported a costutility of US\$75,000 per QALY gained. Two evaluations of extended TAM-AI with letrozole reported favorable cost-utilities in the range of US\$30,000 per QALY gained. Another recent evaluation, by El Ouagari et al. ${ }^{16}$ inalso reported a cost-utility of CDN\$34,000 per QALY gained with extended TAM-LET relative to TAM alone. Evaluations of sequential TAM-AI by Thompson et al. ${ }^{17}$ in, Wilson et al. ${ }^{18}$ in and Lundkvist et al. ${ }^{19}$ in all found TAM-EXE to be a cost-effective strategy compared to TAM alone. Overall, AI strategies appear to be costeffective alternatives to tamoxifen alone for adjuvant hormonal therapy.

C. Skedgel et al. ${ }^{12}$ analysis is the first economic evaluation that compares the incremental cost-utility of upfront, sequential and extended AI strategies using the same Markov model as we used in our analysis, discount rate of $3 \%$ and based on clinical trial results. Their results shows all three AI strategies - upfront, sequential and extended-appear to be cost-effective $(<€ 30,000 /$ QALY gained) alternatives to TAM alone over a 20year horizon. Incremental comparisons among the AI alternatives favored TAM-EXE. Using DFS hazards, TAM-EXE dominated both ANA and TAM-LET, being both less costly and more effective in terms of QALYs gained. Using TTR hazards, the cost-utility of ANA relative to TAM-EXE was unfavorable ( $>€ 30,000 / \mathrm{QALY}$ gained) and TAM-EXE dominated TAM-LET ${ }^{12}$. 
T. Younis et al. ${ }^{13}$ in compared the cost-effectiveness of adjuvant aromatase inhibitor strategies in postmenopausal women with breast cancer according to patients' risk. Their results shows that upfront AI appears to be cost-effective in very high risk patients, while sequential TAM-AI is cost-effective in low and average risk patients.

This study has some limitations: As in all prediction models, this cost-effectiveness analysis relies on key assumptions to reduce the infinite number of possible clinical outcomes to allow a feasible analysis incorporating results of BIG 1-98 analysis. The driving factors in this model were the relative efficacies of letrozole versus tamoxifen, examined and the estimated baseline risks of cancer recurrence and adverse effects. The relative efficacy of both drugs, as reported in the BIG 1-98 trial, was assumed to be constant across cohorts with variable risks of cancer recurrence and to be with constant annual rate. C. Skedgel et al assumed constant annual rate of DFS events 3.37\% in tamoxifen comparison to $2.8 \%$ in anastrazole group. We, therefore, provide a two-way sensitivity analyses across a wide range of HRs for possible future analyses. Lastly, this analysis reflects an Egyptian health care cost perspective, which may not necessarily apply to all other health care systems.

Although most of CEA studies were conducted in the developed countries like USA, Canada.....etc, the developing countries will gain greater benefits from these studies due to their limited resources. So we have to encourage these studies in Egypt to help in proper allocation of the limited health budget indifferent health sectors.

\section{CONCLUSION}

Although letrozole seems to be expensive in comparison to tamoxifen, comprehensive evaluation of the entire drug costs and the gained benefits proved the superiority of letrozole. Awareness of decision makers with this data is of an utmost importance for the direction of budget especially in areas of limited resources such as Egypt.

\section{CORRIGENDUM}

This is a corrected version of the article after adding the name of Dr. Mohamed Elsherbini to the author list and the affiliation of Dr. Mohamed Abdeen. It replaces online the primarily published version as noted in the published corrigendum ("Corrigendum: Abd El Bary et al. Cost Effectiveness Analysis of Letrozole Compared with Tamoxifen as Initial Adjuvant Therapy for Postmenopausal Women with Endocrine Responsive Breast Cancer. Kasr El-Aini J Clin Oncol Nucl Med. 2009;5(3-4):11-17." Res Oncol. 2018; X(X):X-X. DOI: 10.21608/resoncol.2018.6131.1067)

\section{REFERENCES}

1. Meropol NJ, Schulman KA. Cost of cancer care: Issues and implications. J.Clin.Oncol. 2007 10;25(2):180-6.
2. Grusenmeyer PA, Wong YN. Interpreting the economic literature in oncology. J.Clin.Oncol. 2007 Jan 10;25(2):196-202.

3. Devlin N, Parkin D. Does NICE have a cost-effectiveness threshold and what other factors influence its decisions? A binary choice analysis. Health Econ. 2004 May;13(5) :437-52.

4. Ramsey SD. How should we pay the piper when he's calling the tune? On the long-term affordability of cancer care in the United States. J.Clin.Oncol. 2007 Jan $10 ; 25(2): 175-9$.

5. Earle CC, Coyle D, Evans WK. Cost-effectiveness analysis in oncology. Ann.Oncol. 1998 May;9(5):475-82.

6. Hillner BE, Smith TJ. Do the large benefits justify the large costs of adjuvant breast cancer trastuzumab? J.Clin. Oncol. 2007;25(6):611-3.

7. www.worldbank.org. GNI- Gross National Income per capita (PPP\$).

8. Karnon J, Johnston SR, Jones T, Glendenning A. A trialbased cost-effectiveness analysis of letrozole followed by tamoxifen versus tamoxifen followed by letrozole for postmenopausal advanced breast cancer. Ann.Oncol. 2003 Nov;14(11):1629-33.

9. Osborne CK, Zhao H, Fuqua SA. Selective estrogen receptor modulators: Structure, function and clinical use. J.Clin.Oncol. 2000 Sep;18(17):3172-86.

10. Thurlimann B, Keshaviah A, Coates AS, Mouridsen H, Mauriac L, Forbes JF, et al. A comparison of letrozole and tamoxifen in postmenopausal women with early breast cancer. N.Engl.J.Med. 2005 Dec 29;353(26):2747-57.

11. Henning Mouridsen, M.D., of Rigshospitalet in Copenhagen, reported at the San Antonio Breast Cancer Symposium 2008.

12. Skedgel C, Rayson D, Dewar R, Younis T. Costutility of adjuvant hormone therapies with aromatase inhibitors in post-menopausal women with breast cancer: Upfront anastrozole, sequential tamoxifen-exemestane and extended tamoxifen-letrozole. Breast 2007 Jun;16(3):252-61.

13. (Younis T, Rayson D, Dewar R, Skedgel C. Modeling for cost-effective-adjuvant aromatase inhibitor strategies for postmenopausal women with breast cancer. Ann.Oncol. 2007;18(2):293-8.

14. Cocquyt V, Moeremans K, Annemans L, Clarys P, Van Belle S. Long-term medical costs of postmenopausal breast cancer therapy. Ann.Oncol. 2003 Jul;14(7): 105763.

15. Liberato NL, Marchetti M, Barosi G. Cost effectiveness of adjuvant trastuzumab in human epidermal growth factor receptor 2-positive breast cancer. J.Clin.Oncol. 2007;25(6):625-33.

16. Ouagari KE, Karnon J, Delea T, Talbot W, Brandman J. Cost-effectiveness of letrozole in the extended adjuvant treatment of women with early breast cancer. Breast Cancer Res.Treat. 2007;101(1):37-49.

17. Thompson D, Taylor DCA, Montoya EL, Winer EP, Jones SE, Weinstein MC. Cost-effectiveness of switching to exemestane after 2 to 3 years of therapy with tamoxifen in postmenopausal women with early-stage breast cancer. Value Health 2007;10(5):367-76.

18. Wilson K, Wordsworth S, Tabberer M. Exemestane in adjuvant treatment of early breast cancer in postmenopausal women: Results of a UK cost-effectiveness model. Eur.J.Cancer 2006;4(2 Suppl.):151.

19. Lundkvist J, Wilking N, Holmberg S, Jönsson L. Costeffectiveness of exemestane versus tamoxifen as adjuvant therapy for early-stage breast cancer after 2-3 years treatment with tamoxifen in Sweden. Breast Cancer Res. Treat. 2007;102(3):289-99. 